Muro de la Investigación, 2019(2), agosto-diciembre ISSN: 2523-2886

DOI: https://doi.org/10.17162/r-muro-investigaion.v4i2.839

\title{
PROGRAMA “PUKLLASPA RIMASUN" PARA MEJORAR EL LENGUAJE ORAL DE LOS ESTUDIANTES DE NIVEL INICIAL QUECHUA HABLANTES DE 4 Y 5 AÑOS
}

\author{
"Pukllaspa rimasun" Program to improve the oral language of Quechua speakers \\ of 4 and 5 years
}

\author{
Liz Roxana Amiquero Escalante ${ }^{1}$ \\ Universidad Peruana Unión, Perú ${ }^{1}$
}

Recibido: 15 de enero de 2019

Aceptado: 01 de marzo de 2019

\begin{abstract}
Resumen
La presente investigación tuvo como objetivo determinar la efectividad del programa "Pukllaspa rimasun" en la mejora del lenguaje oral en los estudiantes quechua hablantes de 4 y 5 años de la I.E.I $N^{\circ} 785$ de Ubiato del distrito de Kimbiri, La Convención, Cusco, 2018. El tipo de investigación consiste en un enfoque cuantitativo y el diseño es preexperimental. La población estuvo conformada por 10 estudiantes, y la muestra fue por conveniencia; considerando el total de niños matriculados de las edades de 4 y 5 años requerida en esta investigación. A fin de determinar si los puntajes en las variables y dimensiones se aproximaban a una distribución normal se utilizó la prueba de ShapiroWilk, debido a que la muestra fue menor a 50. Para el proceso de análisis estadístico se utilizó el programa Statistical Package of Social Science (SPSS) versión 24.0. Los resultados obtenidos a través del estadígrafo $\mathrm{T}$ de Student y prueba no paramétrica de Wilcoxon demostraron que existieron diferencias significativas antes y después de la aplicación del programa. Debido a que el resultado estadístico del pre test resulta que el $60 \%$ de los estudiantes se encontraban en el nivel necesita mejorar, el $30 \%$ en el nivel normal y el $10 \%$ en el nivel retraso, este tuvo una variación luego de la aplicación del programa, pues en el pos test el $60 \%$ de los estudiantes alcanzaron el nivel normal y el $40 \%$ necesita mejorar, con un nivel de significancia $p=0.000<0.05$. Por lo tanto, se concluye que el programa "Pukllaspa rimasun" fue efectivo en mejorar el lenguaje oral en los estudiantes que participaron en la investigación.
\end{abstract}

Palabras clave: lenguaje oral, fonología, morfología, sintaxis, semántica, pragmática, juegos verbales

\footnotetext{
Abstract

The purpose of this research was to determine the effectiveness of the "Pukllaspa rimasun" program in improving oral language in the Quechua speaking students of 4
} 
and 5 years of the IEI No. 785 of Ubiato of the Kimbiri district, La Convencion, Cusco, 2018 The type of research consists of a quantitative approach and the design is pre-experimental. The population was made up of 10 students, and the sample was for convenience; considering the total number of children enrolled in the ages of 4 and 5 years required in this research. In order to determine if the scores on the variables and dimensions approximated a normal distribution, the Shapiro-Wilk test was used, because the sample was less than 50. For the statistical analysis process the Statistical Package of program was used Social Science (SPSS) version 24.0. The results obtained through Student's T statistic and Wilcoxon's non-parametric test showed that there were significant differences before and after the application of the program. Because the statistical result of the pre-test results that $60 \%$ of the students were in the level needs improvement, $30 \%$ in the normal level and $10 \%$ in the delay level, this had a variation after the application of the program, because in the post test $60 \%$ of the students reached the normal level and $40 \%$ need to improve, with a level of significance $p=0.000<0.05$. Therefore, it is concluded that the "Pukllaspa rimasun" program was effective in improving oral language in students who participated in the research.

Keywords: oral language, phonology, morphology, syntax, semantics, pragmatics, verbal games

\section{Introducción}

El lenguaje oral es la capacidad que tienen los seres humanos para expresar su pensamiento y comunicarse por medio de un sistema de signos vocales o sonidos articulados (Astudillo, (2012). Asimismo, el dominio del lenguaje oral permite la capacidad de comportarse y expresarse de forma diferente en diferentes momentos, porque se asume las normas de conducta social y los hábitos del entorno.

Por su parte, Diaz, (2009) indica que el lenguaje oral es la habilidad humana por excelencia, aquella única característica propia del hombre el cual nos distingue de los animales irracionales y nos humaniza. La habilidad de hablar es el principal fundamento que divide al ser humano de otras especies, y el lenguaje permite manifestar recuerdos, conocimientos, ideas, y deseos, lo que permite comunicarnos de manera directa con nuestro alrededor.

Del mismo modo, Bigas (1996) explica que el lenguaje oral es un instrumento de codificación del pensamiento humano, que permite prepararlo y llevarle a la reflexión así también a la conceptualización. Esta función tiene actos inmensos de repercusiones en el desarrollo cognitivo del hombre, de manera que hace prosperar las habilidades mentales superiores que se encuentran comprometidas en los procesos de enseñanza-aprendizaje, como son la de abstracción, la habilidad de análisis y 
síntesis, la habilidad de delegar situaciones, objetos y personas más allá del "aquí y ahora".

Finalmente, para Calderón Astorga (2004) el lenguaje oral es el medio principal de comunicación humana, mediante el cual se comprenden ideas, se transmiten conocimientos y se expresa verbalmente o por escrito, deseos, sentimientos y acciones; es la respuesta final del proceso de imitación y maduración a través de los estímulos que realizan sus primeros maestros en casa y lo complementan los maestros de educación de primera infancia.

Arce, Chiong, \& Venero (2017) mencionan que el lenguaje oral es un conjunto de reglas muy complejas e intrincadas, lo que exige analizar puntualmente sus dimensiones funcionales, a partir de tres aspectos: aspecto de forma: que contiene la fonología, morfología y sintaxis; el aspecto de contenido: que abarca el significado lingüístico o semántico; y el aspecto de uso: que denomina la interpretación de los enunciados, siendo los componentes que enlazan los sonidos con símbolos en un orden determinado.

Por su parte, Bloom \& Lahey, (1978) detallan el lenguaje como un código con que se comunica el emisor y el receptor; este se establece mediante la acción recíproca de tres sujetos: la forma (fonología, morfología y sintaxis), el contenido (semántica) y el uso del lenguaje (pragmática). Quispe, (2014) expresa que la fonología confirma que existe una relación entre el fonema y contenido del mensaje o sonido que determina el significado de la palabra. Esto debe estimularse en los primeros años del lenguaje del niño, puesto que los juegos verbales, como trabalenguas, adivinanzas, canciones, rimas, segmentación silábica, ayudan a desarrollar la capacidad fonológica del niño.

En otro orden de cosas, Saldaña, (2012) indica que dentro del componente morfológico se ubica la etapa holofrásica del lenguaje (una palabra cada vez) y se basa más que todo en la alternancia de rasgos generales, como el género y el número, hasta otros más complejos como el tiempo verbal, la persona gramatical y los grados del adjetivo.

Por otro lado, Lara, (2015) explica que la sintaxis se encarga del estudio de los sintagmas y oraciones. En el aspecto de la gramática se utiliza un método de reglas para homogenizar las palabras en frases, investiga el orden correcto de las palabras según las reglas que determinan la relación dentro de una oración. 
En cuanto a la semántica, Bloom \& Lahey, (1978) afirma que esta se trata del significado que los objetos poseen en un contexto, los sucesos y de las relaciones que tienen ambos. En esto se aplica el análisis de la significación (expresión o comprensión) ya sean en unidades semánticas (palabras aisladas) o en diferentes contextos como la (expresión de ideas y comprensión de sí misma). Para Quispe (2014) la semántica es el principal componente del lenguaje estrechamente relacionado en la formación de la inteligencia. Gracias a ello permite un análisis especifico del léxico y del proceso de significación de las palabras, la cual es elemental en la preparación de sus capacidades fundamentales que ayuden al niño a comprender y expresar significados de las palabras mediante las unidades lingüísticas.

Además, Quispe, (2014) menciona en cuanto a la pragmática, que es el elemento principal que estudia el aspecto de uso del lenguaje: esta determina la selección de las palabras e interpretación del discurso en diferentes contextos. Es importante evaluar lo que escuchamos en un determinado contexto, ya que debemos sostener como fundamento nuestros aprendizajes previos para poder tener la habilidad de realizar conclusiones. Esto exige entender capacidades básicas para producir un lenguaje objetivo en diferentes situaciones.

\section{Realidad problemática}

En la Institución Educativa Inicial $\mathrm{N}^{\circ} 785$ de Ubiato se observó que los estudiantes quechua hablantes de 4 y 5 años de edad evidencian una gran incapacidad en el uso correcto del lenguaje oral, puesto que un número mínimo de los niños no asisten permanentemente al jardín. Otro de los casos lo constituye los problemas de adaptación social y expresarse en público, cuando el niño es trasladado de una institución a otra repentinamente. Muchos de los padres de familia tienen desconocimiento de la estimulación del lenguaje oral, por ello no trabajan esta capacidad en casa, ni buscan un especialista en el área. Otros menores proceden de hogares disfuncionales, donde hay ausencia de los padres y son acogidos por familiares más cercanos. En el aula, los preescolares demuestran un desarrollo fonológico deficiente para su edad, escasa adquisición de la morfología y la sintaxis, tienen dificultad en la semántica y pragmática para su edad. En este contexto, se sugirió emplear el programa "Pukllaspa rimasun" (se puede traducir "jugar con palabras") con la finalidad de mejorar el lenguaje oral realizando actividades adecuadas que apoyen el desarrollo del lenguaje oral en los niños y niñas. 


\section{Materiales y Métodos}

La presente investigación es de tipo básica, pues busca mejorar el lenguaje oral a través de diferentes estrategias y metodologías planteadas en el programa "Pukllaspa rimasun", con un enfoque cuantitativo para medir su efectividad. El diseño de esta investigación es de tipo pre experimental, porque permite manipular la variable dependiente en estudio "mejorar el lenguaje oral," a fin de producir un efecto esperado a través de la aplicación de la variable independiente "Pukllaspa rimasun." Para ello fue necesario medir las variables en estudio mediante un instrumento de evaluación para obtener los resultados a través de métodos estadísticos. La población y la muestra comprendieron a todos los estudiantes entre las edades de 4 y 5 años (10 niños), de los cuales el, $70 \%$ de los estudiantes quechua hablantes son varones y el 30\% son mujeres. Por otro lado, el $60 \%$ de los estudiantes quechua hablantes dominan el quechua y el $40 \%$ dominan el español; asimismo, el 70\% de los estudiantes quechua hablantes tienen 5 años y el $30 \%$ de los estudiantes tienen 4 años.

Para la recolección de datos se utilizó el Test de la Prueba de Lenguaje Oral (PLON-R), instrumento elaborado por Gloria Aguinaga Ayerra et al. (2004) en Madrid, España, y fue adaptado en Lima Alejandro Dioses Chocano (2006) en la Universidad Mayor de San Marcos. Con ello se evaluó tres dimensiones generales: aspecto de forma de lenguaje, aspecto de contenido, y aspecto de uso; junto con la escala de evaluación (retraso, necesita mejora y normal), antes y después de la aplicación del programa.

Cabe resaltar que el programa "Pukllaspa rimasun" se realizó en la I.E.I. N 785 de Ubiato del distrito de Kimbiri, La Convención, Cusco, 2018, durante tres meses; se desarrolló tres unidades de trabajo, haciendo un total de 18 sesiones de aprendizaje, donde se utilizaron como estrategias las rimas, adivinanzas, cuentos, canciones y juegos.

\section{Resultados}

Los resultados que se muestran en la tabla 1 evidencian que, antes de la aplicación del programa, en la evaluación del pre-test sobre el lenguaje oral, el 60,0\% de los estudiantes quechua hablantes se encontraban en el nivel "necesita mejorar," mientras que el 30,0\% en el nivel "normal," y el 10,0\% en el nivel "retraso." Este resultado cambió después de la ejecución del programa "Pukllaspa rimasun", debido a que en la evaluación del pos-test el $60,0 \%$ de los estudiantes quechua hablantes alcanzaron el nivel "normal," 
el 40,0\% de los estudiantes alcanzaron el nivel "necesita mejorar" y el 0,0\% el nivel "retraso."

Tabla 1

Variable lenguaje oral

\begin{tabular}{lcccc}
\hline \multirow{2}{*}{ Niveles } & \multicolumn{2}{c}{ Antes } & \multicolumn{2}{c}{ Después } \\
\cline { 2 - 5 } & Frecuencia & Porcentaje & Frecuencia & Porcentaje \\
\hline Retraso & 1 & 10,0 & 0 & 0,0 \\
Necesita mejorar & 6 & 60,0 & 4 & 40,0 \\
Normal & 3 & 30,0 & 6 & 60,0 \\
\hline Total & $\mathbf{1 0}$ & $\mathbf{1 0 0 , 0}$ & $\mathbf{1 0}$ & $\mathbf{1 0 0 , 0}$ \\
\hline
\end{tabular}

Los resultados que se muestran en la tabla 2 evidencian que, antes de la aplicación del programa, en la evaluación del pre-test sobre la dimensión del aspecto de forma del lenguaje, el 70,0\% de los estudiantes quechua hablantes se encontraban en el nivel "retraso," el 30,0\% de los estudiantes se encontraban en el nivel "necesita mejorar" y el 0,0\% en el nivel "normal." Este resultado cambió luego de la ejecución del programa "Pukllaspa rimasun," debido a que en la evaluación del pos-test el 60,0\% de los estudiantes alcanzaron el nivel "normal," el 40,0\% alcanzaron en nivel necesita mejorar y el $0,0 \%$ el nivel retraso.

Tabla 2

Dimensión del aspecto de forma del lenguaje oral

\begin{tabular}{lcccc}
\hline \multirow{2}{*}{ Niveles } & \multicolumn{2}{c}{ Antes } & \multicolumn{2}{c}{ Después } \\
\cline { 2 - 5 } & Frecuencia & Porcentaje & Frecuencia & Porcentaje \\
\hline Retraso & 7 & 70,0 & 0 & 0,0 \\
Necesita mejorar & 3 & 30,0 & 4 & 40,0 \\
Normal & 0 & 0,0 & 6 & 60,0 \\
\hline Total & $\mathbf{1 0}$ & $\mathbf{1 0 0 , 0}$ & $\mathbf{1 0}$ & $\mathbf{1 0 0 , 0}$ \\
\hline
\end{tabular}

Los resultados que se muestran en la tabla 3 evidencian que, antes de la aplicación del programa, en la evaluación del pre-test sobre la dimensión del aspecto de contenido del lenguaje, el 50,0\% de los estudiantes quechua hablantes se encontraban en el nivel "normal," mientras que el 40,0\% en el nivel "necesita mejorar" y el 10,0\% en el nivel "retraso." Este resultado cambió luego de la ejecución del programa "Pukllaspa rimasun", 
debido a que en la evaluación del pos-test el 100,0\% de los estudiantes alcanzaron el nivel "normal," el 0,0\% el nivel "necesita mejorar" y el 0,0\% el nivel "retraso."

Tabla 3

Dimensión del aspecto de contenido del lenguaje oral

\begin{tabular}{lcccc}
\hline \multirow{2}{*}{ Niveles } & \multicolumn{2}{c}{ Antes } & \multicolumn{2}{c}{ Después } \\
\cline { 2 - 5 } & Frecuencia & Porcentaje & Frecuencia & Porcentaje \\
\hline Retraso & 1 & 10,0 & 0 & 0,0 \\
Necesita mejorar & 4 & 40,0 & 0 & 0,0 \\
Normal & 5 & 50,0 & 10 & 100,0 \\
\hline Total & $\mathbf{1 0}$ & $\mathbf{1 0 0 , 0}$ & $\mathbf{1 0}$ & $\mathbf{1 0 0 , 0}$ \\
\hline
\end{tabular}

Los resultados que se muestran en la tabla 4 evidencian que, antes de la aplicación del programa, en la evaluación del pre test sobre la dimensión del aspecto de uso del lenguaje, el 60,0\% de los estudiantes quechua hablantes se encontraban en el nivel "necesita mejorar," mientras que el 40,0\% en el nivel "normal" y el 0,0\% en el nivel "retraso." Este resultado cambió luego de la ejecución del programa "Pukllaspa rimasun", debido a que en la evaluación del pos-test el 50,0\% de los estudiantes alcanzaron el nivel "normal," el 50,0\% alcanzaron el nivel "necesita mejorar" y el 0,0\% el nivel "retraso."

Tabla 4

Dimensión del aspecto de uso del lenguaje oral

\begin{tabular}{lcccc}
\hline \multirow{2}{*}{ Niveles } & \multicolumn{2}{c}{ Antes } & \multicolumn{2}{c}{ Después } \\
\cline { 2 - 5 } & Frecuencia & Porcentaje & Frecuencia & Porcentaje \\
\hline Retraso & 0 & 0,0 & 0 & 0,0 \\
Necesita mejorar & 4 & 60,0 & 5 & 50,0 \\
Normal & 4 & 40,0 & 5 & 50,0 \\
& & & & \\
\hline Total & $\mathbf{1 0}$ & $\mathbf{1 0 0 , 0}$ & $\mathbf{1 0}$ & $\mathbf{1 0 0 , 0}$
\end{tabular}

\section{Conclusiones}

El propósito de la presente investigación fue determinar la efectividad del programa "Pukllaspa rimasun" para mejorar el lenguaje oral en los estudiantes quechua 
hablantes de 4 y 5 años de la I.E.I $N^{\circ} 785$ de Ubiato del distrito de Kimbiri, La Convención, Cusco, 2018; "Pukllaspa rimasun"; por lo que a continuación se muestran las siguientes conclusiones:

El programa "Pukllaspa rimasun", es efectivo en la mejora del lenguaje oral de los estudiantes quechua hablantes de 4 y 5 años de la I.E.I N 785 de Ubiato del distrito de Kimbiri, La Convención, Cusco, 2018. Los resultados obtenidos en la evaluación del pre-test sobre el lenguaje oral mostraron que: el 60,0\% de los estudiantes se encontraban en el nivel "necesita mejorar," mientras que el 30,0\% en el nivel "normal" y el 10,0\% en el nivel "retraso." Este resultado tuvo una variación luego de la aplicación del programa, pues en el pos-test el 60,0\% de los estudiantes alcanzaron el nivel "normal" y el 40,0\% el nivel "necesita mejorar."

El programa "Pukllaspa rimasun", es efectivo en la mejora del aspecto formal del lenguaje oral en los estudiantes quechua hablantes de 4 y 5 años de la I.E.I $\mathrm{N}^{\circ} 785$ de Ubiato del distrito de Kimbiri, La Convención, Cusco, 2018. Esto se evidencia en la evaluación del pre-test, donde el 70,0\% de los estudiantes se encontraban en el nivel "retraso," mientras que el 30,0\% se encontraban en el nivel "necesita mejorar." Este resultado tuvo una variación luego de la aplicación del programa, pues en el pos-test el $60,0 \%$ de los estudiantes alcanzaron el nivel "normal" y el 40,0\% el nivel "necesita mejorar."

El programa "Pukllaspa rimasun", es efectivo en la mejora del aspecto de contenido del lenguaje oral en los estudiantes quechua hablantes de 4 y 5 años de la I.E.I № 785 de Ubiato del distrito de Kimbiri, La Convención, Cusco, 2018. Según la evaluación del pre-test, el 50,0\% de los estudiantes se encontraban en el nivel "normal," mientras que el 40,0\% en el nivel "necesita mejorar" y el 10,0\% en el nivel "retraso." Este resultado tuvo una variación luego de la aplicación del programa, pues en el pos-test el 100,0\% de los estudiantes alcanzaron el nivel "normal."

El programa "Pukllaspa rimasun", es efectivo en la mejora del aspecto de uso del lenguaje oral en los estudiantes quechua hablantes de 4 y 5 años de la I.E.I $N^{\circ} 785$ de Ubiato del distrito de Kimbiri, La Convención, Cusco, 2018. En este caso, los resultados demuestran-según el pre-test-que el 60,0\% de los estudiantes se encontraban en el nivel "necesita mejorar," mientras que el 40,0\% en el nivel "normal." Este resultado tuvo una variación luego de la aplicación del programa, porque en el pos-test el 50,0\% de los estudiantes alcanzaron el nivel "normal" y el 50,0\% el nivel "necesita mejorar," lo que significa que en esta dimensión el programa no ayudó significativamente. 


\section{Recomendaciones}

Teniendo en cuenta la efectividad de la investigación para mejorar el lenguaje oral en los estudiantes quechua hablantes de 4 y 5 años de la I.E.I $N^{\circ} 785$ de Ubiato del distrito de Kimbiri, La Convención, Cusco, 2018 se recomienda:

1. Promover el proyecto educativo "Pukllaspa rimasun" para mejorar y fortalecer el lenguaje oral de los niños de educación preescolar.

2. Implementar estrategias metodológicas activas, como los juegos verbales, con el objetivo de desarrollar el lenguaje oral del niño.

3. Promover seminarios y talleres de actualización constantes para las docentes de educación inicial, sobre todo en temas de enseñanza del lenguaje oral.

4. Organizar talleres y charlas contextualizados en la lengua materna a los estudiantes, padres de familia y comunidad en general, a fin que ayuden a mejorar el lenguaje oral bilingüe: quechua y castellano.

5. Los materiales que se utilizan para fortalecer el aprendizaje de los niños del nivel inicial brindados por el Minedu y Digeibira deben estar contextualizados de acuerdo a la realidad lingüística al que pertenecen los estudiantes, con el fin de fortalecer las 47 lenguas originarias, entre ellas andinas y amazónicas, que existen en el Perú.

\section{Referencias}

Arce Zubizarreta, K. L., Chiong Lizano, A. V., \& Venero Gonzales, L. (2017). Evaluación de las propiedades métricas del protocolo de observación del desarrollo de lenguaje para maestras de educación inicial. Pontificia Universidad Católica del Perú.

Astudillo Garcia, R. M. (2012). Lenguaje oral en escolares de primer grado de primaria de zonas urbanas y urbano marginales de Ventanilla - Callao., (53). Retrieved from http://www.upch.edu.pe/faedu/12-pregrado/338-carrera-deeducacion-intercultural-bilingue-beca18

Bigas Salvador, M. (1996). La importancia del lenguaje oral en Educación Infantil. Aula de Innovación Educativa, 46. Retrieved from https://logopedicum.com/wpcontent/uploads/2017/02/la-importancia-del-lenguaje-oral-en-educacioninfantil.pdf

Bloom, L., \& Lahey, M. (1978). Language development and language disorders. Retrieved from file: 
https://academiccommons.columbia.edu/doi/10.7916/D8QZ2GQ5

Calderón Astorga, M. N. (2004). Desarrollo del Lenguaje Oral. (pp. 1-9). Retrieved from

https://www.santafe.gov.ar/index.php/educacion/content/download/174430/858 460/file/Desarrollo del Lenguaje Oral.pdf

Diaz Quintero, M. del M. (2009). "El Lenguaje Oral En El Desarrollo Infantil." $\begin{array}{llll}\text { Desarrollo } & \text { Infantil. } & \text { Retrieved }\end{array}$ https://archivos.csif.es/archivos/andalucia/ensenanza/revistas/csicsif/revista/pdf /Numero_14/MARIA DEL MAR_DIAZ_2.pdf

Lara Castillo, M. P. (2015). El lenguaje oral y la comprensión lectora en los alumnos de primer grado de primaria de una institución educativa estatal y una institución educativa particular del Distrito de San Luis. Universidad Ricardo Palma. Retrieved from http://repositorio.urp.edu.pe/bitstream/handle/urp/734/lara_mp.pdf?sequence=3 \&isAllowed=y

Quispe Román, F. (2014). Efectos del programa “juegos linguísticos” para mejorar el desempeño semántico en los niños de 5 años de una institución educativa del Distrito de Villa el Salvador - Ugel 01. Retrieved from http://tesis.pucp.edu.pe/repositorio/bitstream/handle/123456789/5848/quispe_r oman_flor_efectos_juegos.pdf?sequence $=1$

Saldaña Monago, R. del P. (2012). Programa de cuentos pictográficos para incrementar el lenguaje oral en niños (as) de 3 años de una I.E. del Callao. Retrieved from http://repositorio.usil.edu.pe/bitstream/123456789/1319/1/2012_Saldaña_Progr ama-de-cuentos-pictográficos-para-incrementar-el-lenguaje-oral-en-niñas-de-3años-de-una-institución-educativa-del-Callao.pdf 